Research Article

\title{
Completeness of $b \sim$ Metric Spaces and the Fixed Points of Generalized Multivalued Quasicontractions
}

\author{
Basit Ali, ${ }^{1}$ Mujahid Abbas, ${ }^{2}$ and Manuel de la Sen ${ }^{3}{ }^{3}$ \\ ${ }^{1}$ Department of Mathematics, School of Sciences, University of Management and Technology, C-II, Johar Town, \\ Lahore 54770, Pakistan \\ ${ }^{2}$ Department of Mathematics, Government College University, Lahore 54000, Pakistan \\ ${ }^{3}$ Institute of Research and Development of Processes, University of the Basque Country, Campus of Leioa (Bizkaia), \\ P.O. Box 644- Bilbao, Barrio Sarriena, Leioa 48940, Spain
}

Correspondence should be addressed to Manuel de la Sen; manuel.delasen@ehu.eus

Received 16 May 2020; Accepted 15 August 2020; Published 2 September 2020

Academic Editor: Xiaohua Ding

Copyright $($ C 2020 Basit Ali et al. This is an open access article distributed under the Creative Commons Attribution License, which permits unrestricted use, distribution, and reproduction in any medium, provided the original work is properly cited.

In this article, we present a completeness characterization of $b \sim$ metric space via existence of fixed points of generalized multivalued quasicontractions. The purpose of this paper is twofold: (a) to establish the existence of fixed points of multivalued quasicontractions in the setup of $b \sim$ metric spaces and (b) to establish completeness of a $b \sim$ metric space which is a topological property in nature with existence of fixed points of generalized multivalued quasicontractions. Further, a comparison of our results with comparable results shows that the results obtained herein improve and unify the existing results in the literature applicable to the case where existing results fail.

\section{Introduction and Preliminaries}

In this paper, we consider an important problem in mathematics known as the "completeness problem." Completeness problem is to know under what circumstances the underlying space is complete. Completeness of space guarantees the convergence of all Cauchy sequences. There are some other problems like fixed point problem and variational problem which are equivalent to the completeness problem and readers interested in equivalence of completeness and fixed point problem are referred to a survey article by Cobzaş [1]. Completeness problem in mathematics has correspondence with an important problem known as "the end problem" in behavioral sciences. The end problem is to determine where and when human dynamics defined as succession of positions that starts from an initial position and follows transitions ends somewhere. For details on the completeness problem and the end problem, we refer to [2] and references therein.

On the other hand, the notion of a distance between two objects of an abstract set $Z$ plays a vital role in mathematics and other related disciplines such as routing theory, graph theory, matching problems, and decision-making processes. Due to its significance in solving problems in various disciples, the concept of a distance function (metric) has been generalized in many ways (see [3] and the reference therein). One of such generalizations is the notion of a $b \backsim$ metric introduced by Czerwik [4-6].

In Section 1, we fix the notations along with the provision of necessary tools to prove the main results in this paper. In Section 2, we solve fixed point problem in the context of $b \sim$ metric spaces via more general multivalued quasicontractions. In Section 3, we obtain equivalence between completeness problem and fixed point problem in the context of $b \sim$ metric spaces.

Throughout this article, let $Z$ be a nonempty set and $\mathbb{N}$, $\mathbb{N}_{1}, \mathbb{R}$, and $\mathbb{R}^{+}$denote the sets of positive integers, nonnegative integers, reals, and nonnegative reals, respectively.

Czerwik [4-6] introduced $b \sim$ metric spaces.

Definition 1. A bifunction $\rho: Z \times Z \longrightarrow \mathbb{R}^{+}$is a $b \sim$ metric on $Z$ if there exists a $\kappa \in \mathbb{R}$ with $\kappa \geq 1$ such that for $u, y, z$ in $Z, \rho$ satisfies 
(i) $a_{1}-\rho(u, y)=0$ if $u=y$

(ii) $a_{2}-\rho(u, y)=\rho(y, u)$

(iii) $a_{3}-\rho(u, y) \leq \kappa \rho(u, z)+\kappa \rho(z, y)$

The pair $(Z, \rho)$ is known as $b \sim$ metric space (s) (shortly as $b \sim$ MS (s)) with $b$-metric constant $\kappa$. Clearly, for $\kappa=1$, $(Z, \rho)$ is a metric space, but there are $b \sim$ metrics that are not metrics (see $[4,7,8]$ ).

Let $(Z, \rho)$ be a $b \sim$ MS and $\left\{u_{n}\right\}$ a sequence in $Z$. Then $\left\{u_{n}\right\}$ is a Cauchy sequence if for any given $\varepsilon>0$, there is a $K_{\varepsilon} \in \mathbb{N}$ so that $\rho\left(u_{n}, u_{m}\right)<\varepsilon$ for all $m, n \geq K_{\varepsilon}$, or equivalently, $\left\{u_{n}\right\}$ is Cauchy if

$$
\lim _{n \longrightarrow \infty} \rho\left(u_{n+p}, u_{n}\right)=0,
$$

for all $p \in \mathbb{N}$. Further, $\left\{u_{n}\right\}$ is convergent if there is a $u$ in $Z$ and for any $\varepsilon>0$, there exists $K_{\varepsilon} \in \mathbb{N}$ so that $\rho\left(u_{n}, u\right)<\varepsilon$ for all $n \geq K_{\varepsilon}$, or equivalently, a sequence $\left\{u_{n}\right\}$ in $Z$ converges to $u$ in $Z$ if

$$
\lim _{n \rightarrow \infty} \rho\left(u_{n}, u\right)=0
$$

and we write $u_{n} \longrightarrow u$ as $n \longrightarrow \infty$. A subset $F \subseteq Z$ is closed in $Z$ if for every sequence $\left\{u_{n}\right\}$ in $F$ with $u_{n} \longrightarrow u$ as $n \longrightarrow \infty$, implies $u \in F$ and $F \subseteq Z$ is bounded if

$$
\sup _{z, w \in F} \rho(z, w)<\infty
$$

A $b \sim \operatorname{MS}(Z, \rho)$ is complete if every Cauchy sequence in $Z$ converges. Some topological properties have been discussed in [9]. For instance, a $b$-metric $\rho$ is not continuous in both variables in general. However, if $\rho$ is continuous in one variable, then due to the symmetry property of the $b$-metric $\rho$, it is continuous in the other variable as well. Moreover, a subset

$$
B_{\varepsilon}\left(u_{0}\right)=\left\{u \in Z: \rho\left(u_{0}, u\right)<\varepsilon\right\}
$$

in $(Z, \rho)$ is not an open set (in general) but if $\rho$ is continuous in one variable, then $B_{\varepsilon}\left(u_{0}\right)$ is an open subset in $Z$. Moreover, suppose that the $b$-metric $\rho$ in this paper is continuous in one variable.

The following lemma provides an important tool to prove that a sequence that satisfies certain contractive condition is Cauchy in a $b \sim$ MS.

Lemma 1 (see [10]). If a sequence $\left\{u_{n}\right\}$ in $a b \sim M S(Z, \rho)$ satisfies

$$
\rho\left(u_{n+1}, u_{n+2}\right) \leq r \rho\left(u_{n}, u_{n+1}\right), \quad \forall n \in \mathbb{N},
$$

for some

$$
0 \leq r<\frac{1}{\kappa}
$$

then it is Cauchy sequence in $Z$.

Recently, Suzuki [11] replaced condition (1) with $0<r<1$ and proved the following important lemma in the context of $b \sim$ MSs. It is worth mentioning that the following lemma shows that the criteria to establish Cauchyness of a sequence in $b \sim$ metric and ordinary metric spaces are the same.
Lemma 2 (see [11]). If a sequence $\left\{u_{n}\right\}$ in $a b \sim M S(Z, \rho)$ satisfies

$$
\rho\left(u_{n+1}, u_{n+2}\right) \leq r \rho\left(u_{n}, u_{n+1}\right), \quad \forall n \in \mathbb{N},
$$

for some $r \in[0,1)$, then it is a Cauchy sequence in $Z$.

Let $(Z, \rho)$ be a $b \sim$ MS and $C B(Z)(P(Z))$ the set of nonempty, closed, bounded subsets (nonempty subsets) of $Z$ . For $E, F \in C B(Z)$, the mapping $H_{\rho}$, defined as

$$
H_{\rho}(E, F)=\max \{\delta(E, F), \delta(F, E)\},
$$

is called Hausdorff metric on $C B(Z)$ induced by $\rho$, where

$$
\delta(E, F)=\sup _{u \in E} \rho(u, F), \rho(u, F)=\inf _{w \in F} \rho(u, w) .
$$

The following lemma encompasses some important properties of $b \sim$ MSs that we will use in proving the main results of this article.

Lemma 3 (see $[4-6,8]$ ). For $a b \sim \operatorname{MS}(Z, \rho), u, y \in Z$ and $E, F \in C B(Z)$, the following assertions hold:

$\left(c_{1}\right)\left(C B(Z), H_{\rho}\right)$ is a $b \sim M S$.

$\left(c_{2}\right)$ For all $u \in E, \rho(u, F) \leq H_{\rho}(E, F)$.

$\left(c_{3}\right)$ For all $u, y$ in $Z, \rho(u, E) \leq \kappa \rho(u, y)+\kappa \rho(y, E)$.

(c $\left.c_{4}\right)$ For $t>1$ and $a \in E$, there is $a b \in F$ so that $\rho(a, b) \leq t H_{\rho}(E, F)$.

$\left(c_{5}\right)$ For every $t>0$ and $a \in E$, there is $a b \in F$ so that $\rho(a, b) \leq H_{\rho}(E, F)+t$.

(c $\left.c_{6}\right) a \in \bar{E}=E$ if and only if $\rho(a, E)=0$, where $\bar{E}$ is the closure of $E$ in $(Z, \rho)$.

$\left(c_{7}\right)$ For any sequence $\left\{u_{n}\right\}$ in $Z$,

$$
\begin{gathered}
\rho\left(u_{0}, u_{n}\right) \leq \kappa \rho\left(u_{0}, u_{1}\right)+\kappa^{2} \rho\left(u_{1}, u_{2}\right)+\cdots+\kappa^{n-1} \rho \\
\cdot\left(u_{n-2}, u_{n-1}\right)+\kappa^{n-1} \rho\left(u_{n-1}, u_{n}\right) .
\end{gathered}
$$

Consider a self-mapping on $Z$, that is, $h: Z \longrightarrow Z$ and a multivalued mapping $\mathscr{T}: Z \longrightarrow P(Z)$. The fixed point of $\mathscr{T}$ or $h$ is a point $u$ in $Z$, if $u \in \mathscr{T} u$ or $u=h u$, and we denote the fixed point set of $\mathscr{T}$ or $h$ as $F_{i x}(\mathscr{T})$ or $F_{i x}(h)$.

Regarding the existence of a unique fixed point of $h$, one of the important theorems is Banach contraction principle (BCP) [12]. This principle states that if a self-mapping $h$ on $Z$ (a complete metric space) satisfies

$$
\rho(h u, h y) \leq r \rho(u, y),
$$

for some $0 \leq r<1$, then $F_{i x}(h)$ is singleton. The mapping that satisfies (11) is called a Banach contraction. BCP has been generalized further in more than one directions. For example, Suzuki [13] presented a generalization of BCP that characterized the metric completeness. Kannan $[14,15]$ presented a fixed point result that is independent of BCP but characterizes the metric completeness (see [16]), whereas BCP does not characterize the completeness of underlying metric spaces (see $[17,18])$. Among other generalizations of 
Banach contractions, Ciric [19] introduced a self-mapping $h: Z \longrightarrow Z$ that satisfies

$\rho(h u, h y) \leq r \max \{\rho(u, y), \rho(u, h u), \rho(y, h y), \rho(u, h y), \rho(y, h u)\}$,

for some $0 \leq r<1$ and termed it a quasicontraction. Further, they obtained fixed point results for quasicontractions in orbitally complete metric spaces.

Nadler [20] extended the BCP for multivalued mapping on $Z$ as given below.

Theorem 1. Let $(Z, \rho)$ be a complete metric space and $\mathscr{T}: Z \longrightarrow C B(Z)$ such that

$$
H_{\rho}(\mathscr{T} u, \mathscr{T} y) \leq r \rho(u, y),
$$

for all $u, y \in Z$ and some $r \in[0,1)$; then, $F_{i x}(\mathscr{T})$ is nonempty.

Amini-Harandi [21] generalized Theorem 1 by presenting the following result.

Theorem 2 (see $[21])$. Let $(Z, \rho)$ be a complete metric space and $\mathscr{T}: Z \longrightarrow C B(Z)$. If

$$
\begin{aligned}
H_{\rho}(\mathscr{T} u, \mathscr{T} y) \leq & \max \{\rho(u, y), \rho(u, \mathscr{T} u), \rho(y, \mathscr{T} y), \rho \\
& \cdot(u, \mathscr{T} y), \rho(y, \mathscr{T} u)\},
\end{aligned}
$$

for all $u, y \in Z$ and some $\in\left[0,2^{-1}\right)$, then $F_{i x}(\mathscr{T})$ is nonempty.

Kikkawa and Suzuki [22] generalized Theorem 1 using a function $\xi_{1}:[0,1) \longrightarrow\left(2^{-1}, 1\right]$ defined as

$$
\xi_{1}(t)=\frac{1}{1+t} \text {. }
$$

Theorem 3 (see [22]). Let $(Z, \rho)$ be a complete metric space. If $\mathscr{T}: Z \longrightarrow C B(Z)$ satisfies

$$
\xi_{1}(r) \rho(u, \mathscr{T} u) \leq \rho(u, y) \text { implies } H_{\rho}(\mathscr{T} u, \mathscr{T} y) \leq r \rho(u, y),
$$

for all $u, y \in Z$ and for some $r \in[0,1)$, then $F_{i x}(\mathscr{T})$ is nonempty.

Jovanovic et al. [23] extended BCP in the framework of $b \sim$ MSs.

Theorem 4. Let $(Z, \rho)$ be a complete $b \sim M S$ and $h$ be a selfmapping on $Z$ satisfying

$$
\rho(h u, h y) \leq r \rho(u, y),
$$

for all $u, y \in Z$ and for some $r \in[0,1 / \kappa)$. Then, $F_{i x}(h)$ is singleton.

Dung and Hang [24] replaced the condition $0 \leq r<1 / \kappa$ by $0 \leq r<1$ in the above theorem. They obtained the following version of BCP which shows that BCP can be obtained in $b \sim$ MSs without imposing any additional condition on $r$.

Theorem 5. Let $(Z, \rho)$ be a complete $b \sim M S$ and $h: Z \longrightarrow Z$ be a self-mapping on $Z$ satisfying

$$
\rho(h u, h y) \leq r \rho(u, y),
$$

for all $u, y \in Z$ and for some $r \in[0,1)$. Then, $F_{i x}(h)$ is singleton.

Note that there are fixed point theorems (see [7]) that cannot be transported from metric to $b \sim \mathrm{MSs}$ as in the case of BCP.

Aydi et al. [25] obtained $b \sim$ metric version of Theorem 2 .

Theorem 6 (see [25]). Let $(Z, \rho)$ be a complete $b \sim M S$ and $\mathscr{T}: Z \longrightarrow C B(Z)$. If $\mathscr{T}$ satisfies

$$
\begin{aligned}
& H_{\rho}(\mathscr{T} u, \mathscr{T} y) \leq \max \{\rho(u, y), \rho(u, \mathscr{T} u), \rho(y, \mathscr{T} y), \rho \\
&\cdot(u, \mathscr{T} y), \rho(y, \mathscr{T} u)\}
\end{aligned}
$$

for all $u, y \in Z$ and for some $r \in[0,1)$ with $r<\left(1 / \kappa^{2}+\kappa\right)$, then $F_{i x}(\mathscr{T})$ is nonempty.

Using a function $\xi_{2}:[0,1) \longrightarrow\left(2^{-1}, 1\right]$ defined as $\xi_{2}(r)=1 / 1+\kappa r$, Kutbi et al. [26] presented the next result in $b \sim$ MSs.

Theorem 7 (see [26]). Let $(Z, \rho)$ be a complete $b \sim M S$. If $\mathscr{T}: Z \longrightarrow C B(Z)$ satisfies

$\xi_{2}(r) \rho(u, \mathscr{T} u) \leq \kappa \rho(u, y)$ implies $H_{\rho}(\mathscr{T} u, \mathscr{T} y) \leq r \rho(u, y)$,

for all $u, y \in Z$ and for some $r \in[0,1)$ with $r<\left(1 / \kappa^{2}+\kappa\right)$, then $F_{i x}(\mathscr{T})$ is nonempty.

Let $(Z, \rho)$ be a $b \sim \quad M S, h: Z \longrightarrow Z$ and $\mathscr{T}: Z \longrightarrow C B(Z)$ and let $u, y$ be in $Z$, we use the notations

$$
\begin{aligned}
& N_{\Upsilon}(u, y)=\max \{\rho(u, y), \rho(u, \Upsilon u), \rho(y, \Upsilon y), \rho(u, \Upsilon y), \rho(y, \Upsilon u)\}, \\
& M_{\Upsilon}(u, y)=\max \left\{\rho(u, y), \rho(u, \Upsilon u), \rho(y, \Upsilon y), \rho(u, \Upsilon y), \rho(y, \Upsilon u),(\rho(u, y))^{-1} \rho(u, \Upsilon u) \rho(y, \Upsilon y)\right\},
\end{aligned}
$$

where $\Upsilon \in\{h, \mathscr{T}\}$ and

$$
\Lambda=\left\{\xi: \mathbb{R}^{+} \times \mathbb{R}^{+} \longrightarrow \mathbb{R}: \xi(s, t) \leq \frac{s}{\kappa}-t\right\}
$$

where $\kappa$ is the $b \sim$ metric constant (see [27]).
Recently, Alolaiyan et al. [27] obtained the following theorem for quasicontractions which is a generalization of results in $[7,13,21,25,28,29]$.

Theorem 8 (see [27]). Let $(Z, \rho)$ be a complete $b \sim$ MS. If $\mathscr{T}: Z \longrightarrow C B(Z)$ satisfies 
$\xi(\rho(u, \mathscr{T} u), \rho(u, y)) \leq 0$ implies $H_{\rho}(\mathscr{T} u, \mathscr{T} y) \leq r N_{\mathscr{T}}(u, y)$,

for all $u, y \in Z$ and for some $r \in[0,1)$ with $r<\left(1 / \kappa^{2}+\kappa\right)$, where $\xi \in \Lambda$, then $F_{i x}(\mathscr{T})$ is nonempty.

Theorem 9. Further, in [27], by considering a class $\mathrm{F}$ of $b \sim$ metrics $\rho$ such that for any Cauchy sequence $\left\{u_{n}\right\}$ and any $u$ in $Z$, the sequence $\left\{\rho\left(u, u_{n}\right)\right\}$ is Cauchy in $\mathbb{R}$. One typical example of such $b \sim$ metric $\rho$ is

$$
\rho(u, y)=(v(u, y))^{p},
$$

for $p \geq 1$, where $v$ is a metric on $Z$ (see [27]). For the class $F$ of $b \sim$ metrics, they have obtained the following result.

Theorem 10. Let $(Z, \rho)$ be $a b \sim$ MS such that $\rho \in \mathrm{F}$. For $r \in[0,1)$ with $r<\left(1 / \kappa^{2}+\kappa\right)$, assume that $A_{r, \eta}$ is a class of self-mappings $\mathscr{T}$ of $Z$ that satisfies the following.

(a) For $u, y \in Z$,

$\eta(r) \rho(u, \mathscr{T} u) \leq \kappa \rho(u, y)$ implies $\rho(\mathscr{T} u, \mathscr{T} y) \leq r \rho(u, y)$,

where $\eta:[0,1) \longrightarrow(0,1]$.

Let $B_{r, \eta}$ be the set of mappings $\mathscr{T}$ on $Z$ that satisfies (a). closed.

(b) $\mathscr{T}(Z)$ is denumerable and every subset $D \subseteq \mathscr{T}(Z)$ is

Then, statements (i)-(iii) are equivalent.

(i) $(Z, \rho)$ is complete.

(ii) $F_{i x}(\mathscr{T})$ is nonempty for every $\mathscr{T} \in A_{r, \eta}$ for all $r \in[0,1)$ where $r<\left(1 / \kappa^{2}+\kappa\right)$.

(iii) $F_{i x}(\mathscr{T})$ is nonempty for every $\mathscr{T} \in B_{r, \eta}$ for some $r \in(0,1)$ that satisfies $r<\left(1 / \kappa^{2}+\kappa\right)$.

Motivated by the work in [27], we provide the following definition.

Definition 2. Let $(Z, \rho)$ be a $b \sim$ MS. A mapping $\mathscr{T}: Z \longrightarrow C B(Z)$ is a generalized multivalued Ciric-Suzuki-type (shortly CS-type) quasicontraction if there exists $r \in[0,1)$ with $r<(1 / 2 \kappa)$ such that

$\xi(\rho(u, \mathscr{T} u), \rho(u, y)) \leq 0$ implies $H_{\rho}(\mathscr{T} u, \mathscr{T} y) \leq r M_{\mathscr{T}}(u, y)$, for all $u, y \in Z$, with $u \neq y$, where $\xi \in \Lambda$. If $\mathscr{T}$ is replaced with a self-mapping $h: Z \longrightarrow Z$, then $h$ is generalized CS-type quasicontraction.

In this article, we provide with some new fixed point theorems for generalized multivalued CS-type quasicontractions in $b \sim$ MSs. It is worth noting that the existence of fixed point sets of CS-type quasicontractions further establishes the completeness of underlying $b \sim$ MSs. The authors in [27] obtained a completeness characterization of a specific class of $b \sim$ MSs. We extend their result and present a completeness characterization of any $b \sim$ MS.

\section{Fixed Points of Generalized Multivalued CS - Type Quasicontractions}

The following is the first main result about the fixed points of generalized multivalued CS-type quasicontractions of $b \sim$ MSs.

Theorem 11. Let $(Z, \rho)$ be a complete $b \sim M S$ and $\mathscr{T}: Z \longrightarrow C B(Z)$ be a generalized multivalued CS-type quasicontraction. Then, $F_{i x}(\mathscr{T})$ is nonempty.

Proof. Note that for any $u, y$ in $Z$ with $u \neq y$, we have $M_{\mathscr{T}}(u, y)>0$. As $r<(1 / 2 \kappa)$, we can choose a positive real number $\alpha$ satisfying

$$
\frac{r}{2}+\alpha=\frac{1}{4 \kappa} \text {, that is } r+\alpha=\frac{1}{2}\left(\frac{1}{2 \kappa}+r\right) \text {. }
$$

If $\beta=r+\alpha$, then $0<\beta<1$ and $\alpha>0$. Let $u_{0}$ be any point in $Z$ and $u_{1} \in \mathscr{T} u_{0}$. If $u_{1}=u_{0} \in \mathscr{T} u_{0}, u_{0} \in F_{i x}(\mathscr{T})$. So, assume that $u_{0} \neq u_{1}$.

$$
\xi\left(\rho\left(u_{0}, \mathscr{T} u_{0}\right), \rho\left(u_{0}, u_{1}\right)\right) \leq \frac{1}{\kappa} \rho\left(u_{0}, \mathscr{T} u_{0}\right)-\rho\left(u_{0}, u_{1}\right) \leq 0 .
$$

So, by the given assumption on $\mathscr{T}$, we get that

$$
H_{\rho}\left(\mathscr{T} u_{0}, \mathscr{T} u_{1}\right) \leq r M_{\mathscr{T}}\left(u_{0}, u_{1}\right)
$$

As $\alpha M_{\mathscr{T}}\left(u_{0}, u_{1}\right)>0$, by $\left(c_{5}\right)$ in Lemma 3 , we have $u_{2} \in \mathscr{T} u_{1}$ that satisfies

$$
\rho\left(u_{1}, u_{2}\right) \leq H_{\rho}\left(\mathscr{T} u_{0}, \mathscr{T} u_{1}\right)+\alpha M_{\mathscr{T}}\left(u_{0}, u_{1}\right) .
$$

From (29)-(30), we obtain

$$
\begin{aligned}
\rho\left(u_{1}, u_{2}\right) & \leq H_{\rho}\left(\mathscr{T} u_{0}, \mathscr{T} u_{1}\right)+\alpha M_{\mathscr{T}}\left(u_{0}, u_{1}\right) \\
& \leq r M_{\mathscr{T}}\left(u_{0}, u_{1}\right)+\alpha M_{\mathscr{T}}\left(u_{0}, u_{1}\right)=\beta M_{\mathscr{T}}\left(u_{0}, u_{1}\right) \\
& =\beta \max \left\{\begin{array}{c}
\rho\left(u_{0}, u_{1}\right), \rho\left(u_{0}, \mathscr{T} u_{0}\right), \rho\left(u_{1}, \mathscr{T} u_{1}\right), \rho\left(u_{0}, \mathscr{T} u_{1}\right), \rho\left(u_{1}, \mathscr{T} u_{0}\right), \\
\left(\rho\left(u_{0}, u_{1}\right)\right)^{-1} \rho\left(u_{0}, \mathscr{T} u_{0}\right) \rho\left(u_{1}, \mathscr{T} u_{1}\right)
\end{array}\right\} \\
& \leq \beta \max \left\{\rho\left(u_{0}, u_{1}\right), \rho\left(u_{1}, u_{2}\right), \rho\left(u_{0}, u_{2}\right)\right\} \\
& \leq \beta \max \left\{\rho\left(u_{0}, u_{1}\right), \rho\left(u_{1}, u_{2}\right), \kappa \rho\left(u_{0}, u_{1}\right)+\kappa \rho\left(u_{1}, u_{2}\right)\right\} \\
& \leq \kappa \beta\left(\rho\left(u_{0}, u_{1}\right)+\rho\left(u_{1}, u_{2}\right)\right) .
\end{aligned}
$$


That is,

$$
\rho\left(u_{1}, u_{2}\right) \leq\left(\frac{\kappa \beta}{1-\kappa \beta}\right) \rho\left(u_{0}, u_{1}\right) .
$$

Continuing like this, we obtain a sequence $\left\{u_{n}\right\}$ in $Z$ that satisfies

$$
\begin{gathered}
u_{n+1} \in \mathscr{T} u_{n}, \\
\rho\left(u_{n}, u_{n+1}\right) \leq\left(\frac{\kappa \beta}{1-\kappa \beta}\right) \rho\left(u_{n-1}, u_{n}\right),
\end{gathered}
$$

for all $n \in \mathbb{N}_{1}$. Set $\alpha_{n}=\rho\left(u_{n}, u_{n+1}\right)$; then, from (33), we have

$$
\alpha_{n} \leq \gamma \alpha_{n-1}
$$

where $\gamma=\kappa \beta / 1-\kappa \beta$. As $\kappa \geq 1$ and $r<(1 / 2 \kappa)$, we have

$$
\begin{gathered}
\kappa \beta=\frac{\kappa}{2}\left(\frac{1}{2 \kappa}+r\right)<\frac{1}{2}, \\
\gamma=\frac{\kappa \beta}{1-\kappa \beta}<1 .
\end{gathered}
$$

That is, $\gamma<1$. By Lemma 2, $\left\{u_{n}\right\}$ is a Cauchy sequence in $Z$, and hence

$$
\lim _{n \rightarrow \infty} \rho\left(u_{n}, u\right)=0,
$$

for some $u \in Z$. Now, we show that

$$
\begin{aligned}
\rho\left(u_{n+1}, \mathscr{T} w\right) & \leq H_{\rho}\left(\mathscr{T} u_{n}, \mathscr{T} w\right) \\
& \leq r M_{\mathscr{T}}\left(u_{n}, w\right) \\
& =r \max \left\{\begin{array}{c}
\rho\left(u_{n}, w\right), \rho\left(u_{n}, \mathscr{T} u_{n}\right), \rho(w, \mathscr{T} w), \rho\left(u_{n}, \mathscr{T} w\right), \rho\left(w, \mathscr{T} u_{n}\right), \\
\left(\rho\left(u_{n}, w\right)\right)^{-1} \rho\left(u_{n}, \mathscr{T} u_{n}\right) \rho(w, \mathscr{T} w)
\end{array}\right\} \\
& \leq r \max \left\{\begin{array}{c}
\rho\left(u_{n}, w\right), \rho\left(u_{n}, u_{n+1}\right), \rho(w, \mathscr{T} w), \rho\left(u_{n}, \mathscr{T} w\right), \rho\left(w, u_{n+1}\right), \\
\left(\rho\left(u_{n}, w\right)\right)^{-1} \rho\left(u_{n}, u_{n+1}\right) \rho(w, \mathscr{T} w)
\end{array}\right\},
\end{aligned}
$$

for all $n \geq n_{0}$. By applying the limit as $n \longrightarrow \infty$, we obtain

$$
\rho(u, \mathscr{T} w) \leq r \max \{\rho(u, w), \rho(w, \mathscr{T} w), \rho(u, \mathscr{T} w)\} .
$$

If $\max \{\rho(u, w), \rho(w, \mathscr{T} w), \rho(u, \mathscr{T} w)\}=\rho(u, \mathscr{T} w)$, then we get

$$
\rho(u, \mathscr{T} w) \leq r \rho(u, \mathscr{T} w)<\beta \rho(u, \mathscr{T} w)<\rho(u, \mathscr{T} w),
$$

a contradiction. Consequently, (37) holds for all $w \neq u$.

Now, assume that $z \notin \mathscr{T} z$ for all $z \in Z$. As $r<(1 / 2 \kappa)$, $(1 / 2 r \kappa)-1>0$. Since $\mathscr{T} u$ is nonempty for all $u$ in $Z$, for

$$
\varepsilon=\left(\frac{1}{2 r \kappa}-1\right) \rho(u, \mathscr{T} u)>0,
$$

there exists $a \in \mathscr{T} u$ such that

$$
\rho(u, \mathscr{T} w) \leq r \max \{\rho(u, w), \rho(w, \mathscr{T} w)\},
$$

for all $w \neq u$. From (36), we choose $n_{0} \in \mathbb{N}$ so that

$$
\rho\left(u_{n}, u\right)<\frac{1}{3 \kappa} \rho(u, w),
$$

for all $n \geq n_{0}$ and for all $w \neq u$. Hence,

$$
\begin{aligned}
\xi\left(\rho\left(u_{n}, \mathscr{T} u_{n}\right), \rho\left(u_{n}, w\right)\right) & \leq \frac{1}{\kappa} \rho\left(u_{n}, \mathscr{T} u_{n}\right)-\rho\left(u_{n}, w\right) \\
& \leq \frac{1}{\kappa} \rho\left(u_{n}, u_{n+1}\right)-\rho\left(u_{n}, w\right) \\
& \leq \frac{1}{\kappa}\left(\kappa \rho\left(u_{n}, u\right)+\kappa \rho\left(u, u_{n+1}\right)\right)-\rho\left(u_{n}, w\right) \\
& \leq \frac{2}{3 \kappa} \rho(u, w)-\rho\left(u_{n}, w\right) \\
& =\frac{1}{\kappa}\left(\rho(u, w)-\frac{1}{3} \rho(u, w)\right)-\rho\left(u_{n}, w\right) \\
& \leq \frac{1}{\kappa}\left(\rho(u, w)-\kappa \rho\left(u_{n}, u\right)\right)-\rho\left(u_{n}, w\right) \\
& \leq \frac{1}{\kappa}\left(\kappa \rho\left(u_{n}, w\right)\right)-\rho\left(u_{n}, w\right)=0,
\end{aligned}
$$

for all $n \geq n_{0}$. This further implies that 


$$
\begin{aligned}
H_{\rho}(\mathscr{T} u, \mathscr{T} a) & \leq r M_{\mathscr{T}}(u, a) \\
& \leq r \max \left\{\begin{array}{c}
\rho(u, a), \rho(u, \mathscr{T} u), \rho(a, \mathscr{T} a), \rho(u, \mathscr{T} a), \rho(a, \mathscr{T} u), \\
(\rho(u, a))^{-1} \rho(u, \mathscr{T} u) \rho(a, \mathscr{T} a)
\end{array}\right\} \\
& \leq r \max \left\{\begin{array}{c}
\rho(u, a), \rho(u, a), \rho(a, \mathscr{T} a), \rho(u, \mathscr{T} a), \rho(a, a), \\
(\rho(u, a))^{-1} \rho(u, a) \rho(a, \mathscr{T} a)
\end{array}\right\} \\
& =r \max \{\rho(u, a), \rho(a, \mathscr{T} a), \rho(u, \mathscr{T} a)\} .
\end{aligned}
$$

If

$$
\max \{\rho(u, a), \rho(a, \mathscr{T} a), \rho(u, \mathscr{T} a)\}=\rho(a, \mathscr{T} a),
$$

then

$$
\rho(a, \mathscr{T} a) \leq H_{\rho}(\mathscr{T} u, \mathscr{T} a) \leq r \rho(a, \mathscr{T} a)
$$

which implies that either $a \in \mathscr{T} a$, that is $a \in F_{i x}(\mathscr{T})$, or $\rho(a, \mathscr{T} a)<\rho(a, \mathscr{T} a)$, a contradiction. Consequently, we have

$$
H_{\rho}(\mathscr{T} u, \mathscr{T} a) \leq r \max \{\rho(u, a), \rho(u, \mathscr{T} a)\} .
$$

If

$$
\max \{\rho(u, a), \rho(a, \mathscr{T} a), \rho(u, \mathscr{T} a)\}=\rho(u, \mathscr{T} a),
$$

then (37)-(50) imply that

$$
\begin{aligned}
H_{\rho}(\mathscr{T} u, \mathscr{T} a) & \leq r \rho(u, \mathscr{T} a) \\
& \leq r^{2} \max \{\rho(u, a), \rho(a, \mathscr{T} a)\} \\
& \leq r \max \{\rho(u, a), \rho(a, \mathscr{T} a)\} .
\end{aligned}
$$

Again if $\max \{\rho(u, a), \rho(a, \mathscr{T} a)\}=\rho(a, \mathscr{T} a)$, then either $a \in \mathscr{T} a$ or $\rho(a, \mathscr{T} a)<\rho(a, \mathscr{T} a)$, so

$$
H_{\rho}(\mathscr{T} u, \mathscr{T} a) \leq r \rho(u, a) .
$$

Also, from (37)-(53), we obtain that

$$
\begin{aligned}
\rho(u, \mathscr{T} a) \leq & r \max \{\rho(u, a), \rho(a, \mathscr{T} a)\} \\
& \leq r \max \left\{\rho(u, a), H_{\rho}(\mathscr{T} u, \mathscr{T} a)\right\} \leq r \rho(u, a) .
\end{aligned}
$$

From (45), (53), and (54), we have

$$
\begin{aligned}
\rho(u, \mathscr{T} u) & \leq \kappa \rho(u, \mathscr{T} a)+\kappa H_{\rho}(\mathscr{T} u, \mathscr{T} a) \\
& \leq \kappa r \rho(u, a)+\kappa r \rho(u, a) \\
& =2 \kappa r \rho(u, a)<\rho(u, \mathscr{T} u),
\end{aligned}
$$

a contradiction. Hence, $u \in \mathscr{T} u$.

\section{Remark 1. As}

$$
\frac{1}{\kappa^{2}+\kappa} \leq \frac{1}{2 \kappa} \text { and } N_{\mathscr{T}}(u, y) \leq M_{\mathscr{T}}(u, y)
$$

for all $u, y \in Z, u \neq y$, we obtain Theorem 8 as a particular case of Theorem 11. Further, Theorem 8 is a generalization of Theorems $1-3,6$, and 7 .

Corollary 1. Let $(Z, \rho)$ be a complete $b \sim M S$ and $\mathscr{T}: Z \longrightarrow C B(Z)$. If

$$
\rho(u, \mathscr{T} u) \leq \kappa \rho(u, y) \text { implies } H_{\rho}(\mathscr{T} u, \mathscr{T} y) \leq r M_{\mathscr{T}}(u, y),
$$

for all $u, y \in Z$, such that $u \neq y$, and for some $r \in[0,1 / 2 \kappa)$, then $F_{i x}(\mathscr{T})$ is nonempty.

Corollary 2. Let $(Z, \rho)$ be a complete $b \sim M S$ and $\mathscr{T}: Z \longrightarrow C B(Z)$. If

$$
\rho(u, \mathscr{T} u) \leq \kappa \rho(u, y) \text { implies } H_{\rho}(\mathscr{T} u, \mathscr{T} y) \leq r N_{\mathscr{T}}(u, y) \text {, }
$$

for all $u, y \in Z$ and for some $r \in[0,1 / 2 \kappa)$, then $F_{i x}(\mathscr{T})$ is nonempty.

The next two corollaries are the extensions of Theorem 6 in $b \sim$ MSs as (56) holds for $\kappa \geq 1$.

Corollary 3. Let $(Z, \rho)$ be a complete $b \sim M S$ and $\mathscr{T}: Z \longrightarrow C B(Z)$. If

$$
H_{\rho}(\mathscr{T} u, \mathscr{T} y) \leq r M_{\mathscr{T}}(u, y)
$$

for all $u, y \in Z$, such that $u \neq y$, and for some $r \in[0,1 / 2 \kappa)$, then $F_{i x}(\mathscr{T})$ is nonempty.

Corollary 4. Let $(Z, \rho)$ be a complete $b \sim M S$ and $\mathscr{T}: Z \longrightarrow C B(Z)$. If

$$
H_{\rho}(\mathscr{T} u, \mathscr{T} y) \leq r N_{\mathscr{T}}(u, y),
$$

for all $u, y \in Z$, such that $u \neq y$, and for some $r \in[0,1 / 2 \kappa)$, then $F_{i x}(\mathscr{T})$ is nonempty.

Remark 2. If $\kappa=1$ in Corollary 4, we get Theorem 2 .

Example 1. Let $Z=\left\{u_{1}, u_{2}, u_{3}, u_{4}, u_{5}\right\}$ and $\rho: Z \times Z \longrightarrow \mathbb{R}^{+}$ be defined as 


$$
\begin{aligned}
\rho\left(u_{1}, u_{2}\right) & =\rho\left(u_{1}, u_{3}\right)=5.28, \\
\rho\left(u_{1}, u_{4}\right) & =\rho\left(u_{1}, u_{5}\right)=12, \\
\rho\left(u_{2}, u_{5}\right) & =\rho\left(u_{3}, u_{4}\right)=\rho\left(u_{3}, u_{5}\right)=9, \\
\rho\left(u_{2}, u_{4}\right) & =5.72, \\
\rho\left(u_{2}, u_{3}\right) & =6, \\
\rho\left(u_{4}, u_{5}\right) & =3, \\
\rho(u, u) & =0 \text { and } \rho(u, y)=\rho(y, u), \quad \text { for all } u, y \in Z .
\end{aligned}
$$

As

$$
\rho\left(u_{4}, u_{1}\right)=12 \nless \rho\left(u_{4}, u_{2}\right)+\rho\left(u_{2}, u_{1}\right)=11,
$$

$\rho$ is not a metric on $Z$. For $\kappa \geq(12 / 11)>1, \rho$ is a $b \sim$ metric on $Z$. Let $\xi(s, t)=(s / \kappa)-t \in \Lambda, r=11 / 25$. Then,

$$
\frac{1}{\kappa^{2}+\kappa}=\frac{121}{276} \approx 0.438<r=0.44<0.458 \approx \frac{1}{2 \kappa} \text {. }
$$

Define a mapping $\mathscr{T}: Z \longrightarrow C B(Z)$ by

$$
\mathscr{T} u= \begin{cases}\left\{u_{1}\right\}, & \text { if } u \in\left\{u_{1}, u_{2}, u_{3}\right\}, \\ \left\{u_{2}\right\}, & \text { if } u \in\left\{u_{4}\right\}, \\ \left\{u_{3}\right\}, & \text { if } u \in\left\{u_{5}\right\} .\end{cases}
$$

For all $u, y \in\left\{u_{1}, u_{2}, u_{3}\right\}$,

$$
H_{\rho}(\mathscr{T} u, \mathscr{T} y)=0 \leq r M_{\mathscr{T}}(u, y) .
$$

Note that

$$
\begin{aligned}
& M_{\mathscr{T}}\left(u_{1}, u_{4}\right)=\max \left\{\begin{array}{c}
\rho\left(u_{1}, u_{4}\right), \rho\left(u_{4}, \mathscr{T} u_{4}\right), \rho\left(u_{1}, \mathscr{T} u_{1}\right), \rho\left(u_{4}, \mathscr{T} u_{1}\right), \rho\left(u_{1}, \mathscr{T} u_{4}\right), \\
\left(\rho\left(u_{1}, u_{4}\right)\right)^{-1} \rho\left(u_{4}, \mathscr{T} u_{4}\right) \rho\left(u_{1}, \mathscr{T} u_{1}\right)
\end{array}\right\} \\
& =\max \left\{\begin{array}{c}
\rho\left(u_{1}, u_{4}\right), \rho\left(u_{4}, u_{2}\right), \rho\left(u_{1}, u_{1}\right), \rho\left(u_{4}, u_{1}\right), \rho\left(u_{1}, u_{2}\right), \\
\left(\rho\left(u_{1}, u_{4}\right)\right)^{-1} \rho\left(u_{4}, u_{2}\right) \rho\left(u_{1}, u_{1}\right)
\end{array}\right\} \\
& =\max \{12,5.72,0,12,5.28,0\}=12 \text {, } \\
& M_{\mathscr{T}}\left(u_{1}, u_{5}\right)=\max \left\{\begin{array}{c}
\rho\left(u_{1}, u_{5}\right), \rho\left(u_{5}, \mathscr{T} u_{5}\right), \rho\left(u_{1}, \mathscr{T} u_{1}\right), \rho\left(u_{5}, \mathscr{T} u_{1}\right), \rho\left(u_{1}, \mathscr{T} u_{5}\right), \\
\left(\rho\left(u_{1}, u_{5}\right)\right)^{-1} \rho\left(u_{5}, \mathscr{T} u_{5}\right) \rho\left(u_{1}, \mathscr{T} u_{1}\right)
\end{array}\right\} \\
& =\max \left\{\begin{array}{c}
\rho\left(u_{1}, u_{5}\right), \rho\left(u_{4}, u_{3}\right), \rho\left(u_{1}, u_{1}\right), \rho\left(u_{5}, u_{1}\right), \rho\left(u_{1}, u_{3}\right), \\
\left(\rho\left(u_{1}, u_{5}\right)\right)^{-1} \rho\left(u_{5}, u_{3}\right) \rho\left(u_{1}, u_{1}\right)
\end{array}\right\} \\
& =\max \{12,9,0,12,5.28,0\}=12 \text {. }
\end{aligned}
$$

Further

$$
\begin{aligned}
& M_{\mathscr{T}}\left(u_{2}, u_{4}\right)=\max \left\{\begin{array}{c}
\rho\left(u_{2}, u_{4}\right), \rho\left(u_{4}, \mathscr{T} u_{4}\right), \rho\left(u_{2}, \mathscr{T} u_{2}\right), \rho\left(u_{4}, \mathscr{T} u_{2}\right), \rho\left(u_{2}, \mathscr{T} u_{4}\right), \\
\left(\rho\left(u_{2}, u_{4}\right)\right)^{-1} \rho\left(u_{4}, \mathscr{T} u_{4}\right) \rho\left(u_{2}, \mathscr{T} u_{2}\right)
\end{array}\right\} \\
& =\max \left\{\begin{array}{c}
\rho\left(u_{2}, u_{4}\right), \rho\left(u_{4}, u_{2}\right), \rho\left(u_{2}, u_{1}\right), \rho\left(u_{4}, u_{1}\right), \rho\left(u_{2}, u_{2}\right), \\
\left(\rho\left(u_{2}, u_{4}\right)\right)^{-1} \rho\left(u_{4}, u_{2}\right) \rho\left(u_{2}, u_{1}\right)
\end{array}\right\} \\
& =\max \{5.72,5.72,5.28,12,0,5.28\}=12 \text {, } \\
& M_{\mathscr{T}}\left(u_{2}, u_{5}\right)=\max \left\{\begin{array}{c}
\rho\left(u_{2}, u_{5}\right), \rho\left(u_{5}, \mathscr{T} u_{5}\right), \rho\left(u_{2}, \mathscr{T} u_{2}\right), \rho\left(u_{5}, \mathscr{T} u_{2}\right), \rho\left(u_{2}, \mathscr{T} u_{5}\right), \\
\left(\rho\left(u_{2}, u_{5}\right)\right)^{-1} \rho\left(u_{5}, \mathscr{T} u_{5}\right) \rho\left(u_{2}, \mathscr{T} u_{2}\right)
\end{array}\right\} \\
& =\max \left\{\begin{array}{c}
\rho\left(u_{2}, u_{5}\right), \rho\left(u_{5}, u_{3}\right), \rho\left(u_{2}, u_{1}\right), \rho\left(u_{5}, u_{1}\right), \rho\left(u_{2}, u_{3}\right), \\
\left(\rho\left(u_{2}, u_{5}\right)\right)^{-1} \rho\left(u_{5}, u_{3}\right) \rho\left(u_{2}, u_{1}\right)
\end{array}\right\} \\
& =\max \{9,9,12,12,6,5.28\}=12 \text {. }
\end{aligned}
$$


Moreover,

$$
\begin{aligned}
& M_{\mathscr{T}}\left(u_{3}, u_{4}\right)=\max \left\{\begin{array}{c}
\rho\left(u_{3}, u_{4}\right), \rho\left(u_{4}, \mathscr{T} u_{4}\right), \rho\left(u_{3}, \mathscr{T} u_{3}\right), \rho\left(u_{4}, \mathscr{T} u_{3}\right), \rho\left(u_{3}, \mathscr{T} u_{4}\right), \\
\left(\rho\left(u_{3}, u_{4}\right)\right)^{-1} \rho\left(u_{4}, \mathscr{T} u_{4}\right) \rho\left(u_{3}, \mathscr{T} u_{3}\right)
\end{array}\right\} \\
& =\max \left\{\begin{array}{c}
\rho\left(u_{3}, u_{4}\right), \rho\left(u_{4}, u_{2}\right), \rho\left(u_{3}, u_{1}\right), \rho\left(u_{4}, u_{1}\right), \rho\left(u_{3}, u_{2}\right), \\
\left(\rho\left(u_{3}, u_{4}\right)\right)^{-1} \rho\left(u_{4}, u_{2}\right) \rho\left(u_{3}, u_{1}\right)
\end{array}\right\} \\
& =\max \{9,5.72,5.28,12,6,3.35\}=12 \text {, } \\
& M_{\mathscr{T}}\left(u_{3}, u_{5}\right)=\max \left\{\begin{array}{c}
\rho\left(u_{3}, u_{5}\right), \rho\left(u_{5}, \mathscr{T} u_{5}\right), \rho\left(u_{3}, \mathscr{T} u_{3}\right), \rho\left(u_{5}, \mathscr{T} u_{3}\right), \rho\left(u_{3}, \mathscr{T} u_{5}\right), \\
\left(\rho\left(u_{3}, u_{5}\right)\right)^{-1} \rho\left(u_{5}, \mathscr{T} u_{5}\right) \rho\left(u_{3}, \mathscr{T} u_{3}\right)
\end{array}\right\} \\
& =\max \left\{\begin{array}{c}
\rho\left(u_{3}, u_{5}\right), \rho\left(u_{5}, u_{3}\right), \rho\left(u_{3}, u_{1}\right), \rho\left(u_{5}, u_{1}\right), \rho\left(u_{3}, u_{3}\right), \\
\left(\rho\left(u_{3}, u_{5}\right)\right)^{-1} \rho\left(u_{5}, u_{3}\right) \rho\left(u_{3}, u_{1}\right)
\end{array}\right\} \\
& =\max \{9,9,5.28,12,0,5.28\}=12 \text {. }
\end{aligned}
$$

Hence, for $u \in\left\{u_{1}, u_{2}, u_{3}\right\}$ and $y \in\left\{u_{4}, u_{5}\right\}$,

$$
\begin{aligned}
M_{\mathscr{T}}(u, y) & =12, \\
H_{\rho}(\mathscr{T} u, \mathscr{T} y) & =5.28 .
\end{aligned}
$$

Consequently, for $u \in\left\{u_{1}, u_{2}, u_{3}\right\}$ and $y \in\left\{u_{4}, u_{5}\right\}$, the implication

$\xi(\rho(u, \mathscr{T} u), \rho(u, y)) \leq 0$ implies $H_{\rho}(\mathscr{T} u, \mathscr{T} y) \leq r M_{\mathscr{T}}(u, y)$

holds true. For $u, y \in\left\{u_{4}, u_{5}\right\}, u \neq y$, we get

$$
\begin{aligned}
& \xi\left(\rho\left(u_{4}, \mathscr{T} u_{4}\right), \rho\left(u_{4}, u_{5}\right)\right)=\kappa^{-1} \rho\left(u_{4}, \mathscr{T} u_{4}\right)-\rho\left(u_{4}, u_{5}\right) \approx 2.24>0, \\
& \xi\left(\rho\left(u_{5}, \mathscr{T} u_{5}\right), \rho\left(u_{5}, u_{4}\right)\right)=\kappa^{-1} \rho\left(u_{5}, \mathscr{T} u_{5}\right)-\rho\left(u_{4}, u_{5}\right) \approx 5.25>0 .
\end{aligned}
$$

Hence, all the assumptions in Theorem 11 are satisfied.

Now based on the above calculations, we present a comparison among Theorem 11 and some results from the literature.
(1) If $u=u_{1}$, and $y=u_{4}$, then

$$
\begin{aligned}
\xi\left(\rho\left(u_{1}, \mathscr{T} u_{1}\right), \rho\left(u_{1}, u_{4}\right)\right)= & \kappa^{-1} \rho\left(u_{1}, \mathscr{T} u_{1}\right) \\
& -\rho\left(u_{1}, u_{4}\right)=-12 \leq 0,
\end{aligned}
$$

but

$$
\begin{aligned}
H_{\rho}\left(\mathscr{T} u_{1}, \mathscr{T} u_{4}\right) & =\rho\left(u_{1}, u_{2}\right)=5.28 \not 12 r=r M_{\mathscr{T}}\left(u_{1}, u_{4}\right) \\
& =r N_{\mathscr{T}}\left(u_{1}, u_{4}\right),
\end{aligned}
$$

for any $r<0.438 \approx 1 / \kappa^{2}+\kappa$, so Theorem 8 (a main result in [27]) cannot be applied in this example. Hence, Theorem 8 is a generalization of Theorem 8 .

(2) Now, $H_{\rho}\left(\mathscr{T} u_{4}, \mathscr{T} u_{5}\right)=\rho\left(u_{2}, u_{3}\right)=6$ and

$$
\left.\begin{array}{rl}
M_{\mathscr{T}}\left(u_{4}, u_{5}\right) & =\max \left\{\begin{array}{c}
\rho\left(u_{4}, u_{5}\right), \rho\left(u_{4}, \mathscr{T} u_{4}\right), \rho\left(u_{5}, \mathscr{T} u_{5}\right), \rho\left(u_{4}, \mathscr{T} u_{5}\right), \rho\left(u_{5}, \mathscr{T} u_{4}\right) \\
\left(\rho\left(u_{4}, u_{5}\right)\right)^{-1} \rho\left(u_{4}, \mathscr{T} u_{4}\right) \rho\left(u_{5}, \mathscr{T} u_{5}\right)
\end{array}\right\} \\
& =\max \left\{\begin{array}{c}
\rho\left(u_{4}, u_{5}\right), \rho\left(u_{4}, u_{2}\right), \rho\left(u_{5}, u_{3}\right), \rho\left(u_{4}, u_{3}\right), \rho\left(u_{5}, u_{2}\right), \\
\left(\rho\left(u_{4}, u_{5}\right)\right)^{-1} \rho\left(u_{4}, u_{2}\right) \rho\left(u_{5}, u_{3}\right)
\end{array}\right\} \\
& =\max \left\{3,5.72,9,9,9, \frac{(5.72)(9)}{3}\right\}=17.16
\end{array}\right\}
$$


On the other hand,

$$
H_{\rho}\left(\mathscr{T} u_{4}, \mathscr{T} u_{5}\right)=6 \not 9 r=r N_{\mathscr{T}}\left(u_{4}, u_{5}\right),
$$

for any $r<0.438 \approx 1 / \kappa^{2}+\kappa$. Hence, Theorem 6 is not applicable in this example. So, Theorem 11 is a proper generalization of Theorem 6 which itself is a generalization of Theorems 1 and 2 and Theorem 1 of [7].

We give another example to show the fact that Theorem 11 is applicable to certain cases where several existing results are not.

Example 2. Let $Z=\left\{u_{1}, u_{2}, u_{3}\right\}$. Suppose that bifunction $\rho: Z \times Z \longrightarrow \mathbb{R}^{+}$is given by

$$
\begin{aligned}
\rho\left(u_{1}, u_{2}\right) & =4, \\
\rho\left(u_{1}, u_{3}\right) & =1.4, \\
\rho\left(u_{2}, u_{3}\right) & =1.6, \\
\rho(u, u) & =0, \\
\rho(u, y) & =\rho(y, u),
\end{aligned}
$$

for all $u, y \in Z$.

As $4=\rho\left(u_{2}, u_{1}\right) \not \rho\left(u_{2}, u_{3}\right)+\rho\left(u_{3}, u_{1}\right)=3, \rho$ is not a metric on $Z$. For $\kappa \geq(4 / 3)>1, \rho$ is a $b \sim$ metric on $Z$. Consider $\mathscr{T}: Z \longrightarrow C B(Z)$ defined as

$$
\mathscr{T} u= \begin{cases}\left\{u_{1}, u_{3}\right\}, & \text { if } u \in\left\{u_{1}, u_{3}\right\}, \\ \left\{u_{1}\right\}, & \text { if } u \in\left\{u_{2}\right\} .\end{cases}
$$

If we consider $\xi(s, t)=(s / \kappa)-t \in \Lambda$ and $r=0.35$, then

$$
\frac{1}{\kappa^{2}+\kappa}=\frac{9}{28} \approx 0.32<r=0.35<0.375=\frac{3}{8}=\frac{1}{2 \kappa} \text {. }
$$

For $u, y \in\left\{u_{1}, u_{3}\right\}$, we have

$$
H_{\rho}(\mathscr{T} u, \mathscr{T} y)=0 \leq r M_{\mathscr{T}}(u, y) .
$$

Hence, for $u \in\left\{u_{1}, u_{3}\right\}$ and $y=u_{2}$,

$$
\begin{aligned}
H_{\rho}(\mathscr{T} u, \mathscr{T} y) & =1.4, \\
M_{\mathscr{T}}(u, y) & =N_{\mathscr{T}}(u, y)=4 \operatorname{as} \rho(u, \mathscr{T} u) \\
& =0, \text { and } \rho(y, \mathscr{T} y)=4 .
\end{aligned}
$$

That is,

$$
H_{\rho}(\mathscr{T} u, \mathscr{T} y)=1.4 \leq 1.4=r M_{\mathscr{T}}(u, y) .
$$

Hence,

$\xi(\rho(u, \mathscr{T} u), \rho(u, y)) \leq 0$ implies $H_{\rho}(\mathscr{T} u, \mathscr{T} y) \leq r M_{\mathscr{T}}(u, y)$,

for all $u, y \in Z, u \neq y$. That is, all the assumptions of Theorem 11 are met. If $u=u_{2}, y=u_{3}$, then

$$
\begin{gathered}
\xi_{2}(r) \rho\left(u_{3}, \mathscr{T} u_{3}\right)=0 \leq \kappa \rho\left(u_{3}, u_{2}\right)=2, \\
H_{\rho}\left(\mathscr{T} u_{3}, \mathscr{T} u_{2}\right)=\rho\left(u_{1}, u_{3}\right)=1.4 .
\end{gathered}
$$

But $H_{\rho}\left(\mathscr{T} u_{3}, \mathscr{T} u_{2}\right)=1.4 \not 1.6 r=r \rho\left(u_{3}, u_{2}\right)$ for any $r<9 / 28=1 / \kappa^{2}+\kappa$. Hence, Theorem 7 cannot be applied in this situation. So, Theorem 11 is a proper generalization of Theorem 7 which generalizes Theorems 1 and 3 .

Corollary 5. Let $(Z, \rho)$ be a complete $b \sim M S$ and $h: Z \longrightarrow Z$ be a generalized CS-type quasicontraction. Then, $F_{i x}(h)$ is singleton.

Proof. From Theorem 11, there exists $u_{0} \in F_{i x}(h)$. To prove the uniqueness, assume $u_{0}, y_{0} \in F_{i x}(h)$ with $u_{0} \neq y_{0}$. As

$$
\begin{aligned}
\xi\left(\rho\left(u_{0}, h u_{0}\right), \rho\left(u_{0}, y_{0}\right)\right) & \leq \frac{1}{\kappa} \rho\left(u_{0}, h u_{0}\right)-\rho\left(u_{0}, y_{0}\right) \\
& =-\frac{1}{\kappa} \rho\left(u_{0}, y_{0}\right) \leq 0,
\end{aligned}
$$

we have

$$
\begin{aligned}
\rho\left(u_{0}, y_{0}\right) & =\rho\left(h u_{0}, h y_{0}\right) \leq r M_{h}\left(u_{0}, y_{0}\right) \\
& =r \max \left\{\begin{array}{c}
\rho\left(u_{0}, y_{0}\right), \rho\left(u_{0}, h u_{0}\right), \rho\left(y_{0}, h y_{0}\right), \rho\left(u_{0}, h y_{0}\right), \rho\left(y_{0}, h u_{0}\right), \\
\left(\rho\left(u_{0}, y_{0}\right)\right)^{-1} \rho\left(u_{0}, h u_{0}\right) \rho\left(y_{0}, h y_{0}\right)
\end{array}\right\} \\
& =r \rho\left(u_{0}, y_{0}\right)<\rho\left(u_{0}, y_{0}\right) \text { as } r<1,
\end{aligned}
$$

a contradiction. Hence, $F_{i x}(h)$ is singleton.

Corollary 6. Let $(Z, \rho)$ be a complete $b \sim M S$ and $h: Z \longrightarrow Z$. If

$$
\rho(u, h u) \leq \kappa \rho(u, y) \text { implies } \rho(h u, h y) \leq r \rho(u, y),
$$

for all $u, y \in Z$ and for some $r \in[0,1 / 2 \kappa)$, then $F_{i x}(h)$ is singleton.
Corollary 7. Let $(Z, \rho)$ be a complete $b \sim M S$ and $h: Z \longrightarrow Z$. If there exists $\xi \in \Lambda$ and $r \in[0,1)$ with $r<1 / 2 \kappa$ such that

$$
\xi(\rho(u, h u), \rho(u, y)) \leq 0 \text { implies } \rho(h u, h y) \leq r \rho(u, y),
$$

for all $u, y \in Z$, then $F_{i x}(h)$ is singleton.

Proof. It follows from Corollary 5. 
Corollary 8. Let $(Z, \rho)$ be a complete $b \sim M S$ and $h: Z \longrightarrow Z$. If there exists $r \in[0,1)$ with $r<1 / 2 \kappa$ such that $\eta(r) \rho(u, h u) \leq \kappa \rho(u, y)$ implies $\rho(h u, h y) \leq r \rho(u, y)$,

for all $u, y \in Z$, where $\eta:[0,1) \longrightarrow(0,1]$, then $F_{i x}(h)$ is singleton.

Proof. Consider

$$
\xi(s, t)=\frac{\eta(r)}{\kappa} s-t \leq \frac{s}{\kappa}-t .
$$

Hence, $\xi \in \Lambda$. If $s=\rho(u, h u)$ and $t=\rho(u, y)$, then

$$
\xi(\rho(u, h u), \rho(u, y))=\frac{\eta(r)}{\kappa} s-t \leq 0 .
$$

Hence, the result follows from Corollary 7.

\section{Completeness Characterization of $\boldsymbol{b}$ Metric Spaces}

In this section, we discuss the completeness of $b \sim$ MSs via fixed point results.

In 1959, Connel [17] studied some important topological properties of fixed point sets of mappings of metric spaces and presented an example of a separable and locally contractible incomplete metric space $Z$ such that every Banach contraction on $Z$ has a fixed point. This shows that the converse of BCP does not hold true (see [18]). For the sake of completeness and convenience, we present the example initially given in ([17], Example 3).

Example 3. Let $f: Z \longrightarrow Z$ be any Banach contraction on $Z$, where

$I_{0}=[(0,0),(1,0)]$, a horizontal line segment joining $(0,0)$ to $\left.(1,0)\right)$, $I_{n}=\left[\left(n^{-1}, 0\right),\left(n^{-1}, 1\right)\right], \quad n \in \mathbb{N}$, (vertical line segments),

$$
Z=\bigcup_{n \in \mathbb{N}_{1}} I_{n} \text {. }
$$

We prove that $F_{i x}(f)$ is nonempty. Assume that $F_{i x}(f)$ is empty. Let $f_{0}$ be the restriction of $f$ on $I_{0}$. Define

$$
\begin{aligned}
\pi: f_{0}\left(I_{0}\right) & \longrightarrow I_{0}, \\
h=\pi^{\circ} f_{0}: I_{0} & \longrightarrow I_{0} .
\end{aligned}
$$

Since $h$ is a continuous mapping on $I_{0}$, there is $(p, 0) \in F_{i x}(h)$.

Claim: $p=1 / k$ for some integer $k$. If $p \neq 1 / k$ for all integers $k$, then

$$
h(p, 0)=\left(\pi^{\circ} f_{0}\right)(p, 0)=(p, 0) .
$$

Moreover,

$$
f_{0}(p, 0) \notin I_{n}, \quad \text { for all } n \in \mathbb{N} .
$$

Otherwise, if $f_{0}(p, 0) \in I_{n_{1}}$ for some $n_{1} \in \mathbb{N}$, then

$$
f_{0}(p, 0)=\left(\frac{1}{n_{1}}, y\right),
$$

for some $y \in(0,1)$. Consequently, we get

$$
h(p, 0)=\pi\left(f_{0}(p, 0)\right)=\pi\left(\frac{1}{n_{1}}, y\right)=\left(\frac{1}{n_{1}}, 0\right) .
$$

But $h(p, 0)=(p, 0)$ implies that $p=1 / n_{1}$, a contradiction, as $p \neq 1 / k$ for all integers $k$. Hence, (94) holds. Thus, $f_{0}(p, 0) \in I_{0}$ and

$$
\pi\left(f_{0}(p, 0)\right)=f_{0}(p, 0) .
$$

From (93)-(97), we have

$$
f_{0}(p, 0)=(p, 0),
$$

a contradiction as $f_{0}$ has no fixed point. Hence, $p=1 / k$ for some integer $k$. As $F_{i x}(f)$ is empty,

$$
f\left(\frac{1}{k}, 0\right)=\left(\frac{1}{k}, y\right) \text {, }
$$

for some $0<y \leq 1$. Set

$$
A=\left\{y \text { : there is a } z \text { so that } f\left(\frac{1}{k}, y\right)=\left(\frac{1}{k}, z\right), z>y\right\} .
$$

If $t=\sup A$, there is a sequence $\left\{t_{n}\right\}$ in $A$ such that $t_{n} \longrightarrow t$ as $n \longrightarrow \infty$ and there is $s \in[0,1]$ such that

$$
f\left(\frac{1}{k}, t\right)=\left(\frac{1}{k}, s\right) \text {. }
$$

We claim that $t=s$.

3.1. Case (I). If $s<t$, then there exists $s_{1}$ such that $s<s_{1}<t$ and $t_{n} \in\left(s_{1}, t\right)$ eventually. As

$$
\begin{aligned}
f\left(\frac{1}{k}, t_{n}\right) \longrightarrow & f\left(\frac{1}{k}, t\right)=\left(\frac{1}{k}, s\right) \text { as } n \longrightarrow \infty, \\
f\left(\frac{1}{k}, t_{n}\right) & \in\left\{\left(\frac{1}{k}, z\right): z \in\left(0, s_{1}\right)\right\},
\end{aligned}
$$

eventually. This implies

$$
f\left(\frac{1}{k}, t_{n}\right)=\left(\frac{1}{k}, u_{n}\right) .
$$

But $0<u_{n}<s_{1} \leq t_{n}$ eventually, a contradiction to $t_{n} \in A$.

3.2. Case (II). If $t<s$, then there is $s_{1}$ such that $t<s_{1}<s$ and $t_{n} \in\left(t, s_{1}\right)$ eventually. Since

$$
\begin{aligned}
f\left(\frac{1}{k}, t_{n}\right) \longrightarrow & f\left(\frac{1}{k}, t\right)=\left(\frac{1}{k}, s\right) \text { as } n \longrightarrow \infty, \\
f\left(\frac{1}{k}, t_{n}\right) & \in\left\{\left(\frac{1}{k}, z\right): z \in\left(s_{1}, s\right)\right\},
\end{aligned}
$$

eventually and 


$$
f\left(\frac{1}{k}, t_{n}\right)=\left(\frac{1}{k}, u_{n}\right)
$$

But $t<t_{n}<s_{1}<u_{n}<s$ (eventually). Hence, there exists an integer $n_{0}$ such that

$$
f\left(\frac{1}{k}, t_{n_{0}}\right)=\left(\frac{1}{k}, u_{n_{0}}\right) .
$$

But $t<t_{n_{0}}<s_{1}<u_{n_{0}}<s$. This implies $t_{n_{0}} \in A$, a contradiction as $t_{n_{0}}>t=\sup A$. Hence, $s=t$, and

$$
f\left(\frac{1}{k}, t\right)=\left(\frac{1}{k}, t\right)
$$

That is, $F_{i x}(f)$ is nonempty but $Z$ is not complete as it is not closed.

Remark 3. As every metric is a $b \sim$ metric, Theorem 5 and Example 3 show that BCP in $b \sim$ MSs does not characterize the completeness of underlying $b \sim$ MS.

Park and Rhoads [30] commented on completeness characterization of metric spaces and Alolaiyan et al. [27] presented those comments in the context of $b \sim$ MSs as follows. "Let $(Z, \rho)$ be a $b \sim$ MS and $B$ be a class of mappings of $Z$ such that if any map in $B$ has a fixed point, then $Z$ is complete. Let $A$ be a class of mappings of $Z$ containing $B$ such that completeness of $Z$ implies the existence of fixed point of any map in $A$. "

Theorem 12 (see $[27,30])$. If $(Z, \rho)$ is a $b \sim M S$, then $Z$ is complete if $F_{i x}(T)$ is nonempty for any mapping in $T \widehat{I} A$.

Now, we provide a characterization of completeness of $b \sim$ MS.

Theorem 13. Let $(Z, \rho)$ be $a b \sim M S$ and $\mathscr{T}: Z \longrightarrow Z$, such that

(a) For $x, y \in Z$,

$\eta(r) \rho(x, \mathscr{T} x) \leq \kappa \rho(x, y)$ implies $\rho(\mathscr{T} x, \mathscr{T} y) \leq r \rho(x, y)$,

where $r \in[0,1 / 2 \kappa)$ and $\eta:[0,1) \longrightarrow(0,1]$.

(b) For $x, y \in Z$,

$\eta(r) \rho(x, \mathscr{T} x) \leq \kappa \rho(x, y)$ implies $\rho(\mathscr{T} x, \mathscr{T} y) \leq r \rho(x, y)$,

where $r \in\left[0,1 / \kappa^{2}+\kappa\right)$ and $\eta:[0,1) \longrightarrow(0,1]$.

(c) $\mathscr{T}(Z)$ is denumerable.

(d) Every $M \subseteq \mathscr{T}(Z)$ is closed.

Let $A_{r, \eta}$ be a class of mappings $\mathscr{T}$ of $Z$ that satisfies (a), $A_{r, \eta}^{*}$ be a class of mappings $\mathscr{T}$ of $Z$ that satisfies (b), and $B_{r, \eta}$ be a set of mappings $\mathscr{T}$ of $Z$ that satisfies (b), (c), and (d). Then, statements (1)-(4) are equivalent:

(1) $b \sim \operatorname{MS}(Z, \rho)$ is complete.

(2) $F_{i x}(\mathscr{T})$ is nonempty for every $\mathscr{T} \in A_{r, \eta}$ for all $r \in[0,1)$ with $r<1 / 2 \kappa$.

(3) $F_{i x}(\mathscr{T})$ is nonempty for every $\mathscr{T} \in A_{r, \eta}^{*}$ for all $r \in[0,1)$ with $r<1 / \kappa^{2}+\kappa$.

(4) $F_{i x}(\mathscr{T})$ is nonempty for every $\mathscr{T} \in B_{r, \eta}$ for some $r \in(0,1)$ with $r<1 / \kappa^{2}+\kappa$.

Proof. By Corollary 8, (6) implies (11).

As $1 / \kappa^{2}+\kappa \leq 1 / 2 \kappa, A_{r, \eta}^{*} \subseteq A_{r, \eta}$. Hence, (6) implies (29). Since $B_{r, \eta} \subseteq A_{r, \eta}^{*}$, (29) implies (30). (30) implies (6), and assume on contrary that $(30)$ holds but $(Z, \rho)$ is incomplete. That is, there is a sequence $\left\{u_{n}\right\}$ which is Cauchy but does not converge. Define $g: Z \longrightarrow[0, \infty)$ as

$$
g(x)=\lim _{n \longrightarrow \infty} \sup \rho\left(x, u_{n}\right),
$$

for $x \in Z$. As $\left\{u_{n}\right\}$ is Cauchy, for $\varepsilon=1 / \kappa>0$, we can choose $m_{\varepsilon} \in \mathbb{N}$ so that for all $k \in \mathbb{N}$,

$$
\rho\left(u_{m_{\varepsilon}}, u_{m_{\varepsilon}+k}\right) \leq \frac{1}{\kappa}
$$

Hence, for all $k \in \mathbb{N}$, we get

$$
\rho\left(x, u_{m_{\varepsilon}+k}\right) \leq \kappa \rho\left(x, u_{m_{\varepsilon}}\right)+\kappa \rho\left(u_{m_{\varepsilon}}, u_{m_{\varepsilon}+k}\right) \leq \kappa \rho\left(x, u_{m_{\varepsilon}}\right)+1 \text {, }
$$

which implies that the sequence $\left\{\rho\left(x, u_{n}\right)\right\}$ is bounded in $\mathbb{R}$ for every $x \in Z$. This further implies that the function $g$ is well defined. Further, $g(x)>0$ for all $x$ in $Z$. For $\varepsilon>0$, there exists $K_{\varepsilon} \in \mathbb{N}$ such that for all $k \in \mathbb{N}$,

$$
\rho\left(u_{n}, u_{n+k}\right)<\varepsilon
$$

for all $n \geq K_{\varepsilon}$. Hence, we get for all $k \in \mathbb{N}$,

$$
0 \leq g\left(u_{n}\right)=\lim _{k \longrightarrow \infty} \sup \rho\left(u_{n}, u_{n+k}\right)<\varepsilon,
$$

for all $n \geq K_{\varepsilon}$. That is,

$$
\lim _{n \longrightarrow \infty} g\left(u_{n}\right)=0 .
$$

From (118), for every $x \in Z$, there exists $v \in \mathbb{N}$ such that

$$
g\left(u_{v}\right) \leq\left(\frac{r \eta(r)}{3 \kappa^{4}+r \kappa \eta(r)}\right) g(x) .
$$

If $\mathscr{T}(x)=u_{v}$, then

$$
g(\mathscr{T} x) \leq\left(\frac{r \eta(r)}{3 \kappa^{4}+r \kappa \eta(r)}\right) g(x) \text { and } \mathscr{T} x \in\left\{u_{n}: n \in \mathbb{N}\right\},
$$

for all $x \in Z$. From (120), we have $g(\mathscr{T} x)<g(x)$; hence, $\mathscr{T} x \neq x$ for all $x \in Z$. That is, $F_{i x}(\mathscr{T})$ is empty. As $\mathscr{T}(Z) \subset\left\{u_{n}: n \in \mathbb{N}\right\},(\mathrm{c})$ holds. Note that (d) holds as well. Further, $g$ satisfies 


$$
\begin{array}{ll}
g(x)-\kappa g(y) \leq \kappa \rho(x, y), & \text { for all } x, y \in Z, \\
g(y)-\kappa g(x) \leq \kappa \rho(x, y), & \text { for all } x, y \in Z, \\
g(x)-\kappa g(\mathscr{T} x) \leq \kappa \rho(x, \mathscr{T} x), & \text { for all } x \in Z, \\
\rho(\mathscr{T} x, \mathscr{T} y) \leq \kappa g(\mathscr{T} x)+\kappa g(\mathscr{T} y), & \text { for all } x, y \in Z .
\end{array}
$$

Now fix $x, y \in Z$ such that

$$
\eta(r) \rho(x, \mathscr{T} x) \leq \kappa \rho(x, y)
$$

We need to show that (111) holds. Observe that

$$
\left\{\begin{array}{l}
\rho(x, y) \geq \frac{\eta(r)}{\kappa} \rho(x, \mathscr{T} x) \\
\geq \frac{\eta(r)}{\kappa^{2}}(g(x)-\kappa g(\mathscr{T} x)) \\
\geq \frac{\eta(r)}{\kappa^{2}}\left(1-\frac{r \eta(r)}{3 \kappa^{3}+r \eta(r)}\right) g(x) \\
=\frac{3 \kappa \eta(r)}{3 \kappa^{3}+r \eta(r)} g(x) .
\end{array}\right.
$$

We have two cases.

Case (1) Suppose $g(y) \geq 2 \kappa g(x)$; then,

$$
\begin{aligned}
\rho(\mathscr{T} x, \mathscr{T} y) & \leq \kappa g(\mathscr{T} x)+\kappa g(\mathscr{T} y) \\
& \leq \frac{r \eta(r)}{3 \kappa^{3}+r \eta(r)} g(x)+\frac{r \eta(r)}{3 \kappa^{3}+r \eta(r)} g(y) \\
& \leq \frac{r}{3 \kappa}(g(x)+g(y))+\frac{2 r}{3 \kappa}(g(y)-2 \kappa g(x)) \\
& =\frac{r}{3}\left(\frac{1}{\kappa} g(x)+\frac{1}{\kappa} g(y)+\frac{2}{\kappa} g(y)-4 g(x)\right) \\
& \leq \frac{r}{3}\left(\frac{3}{\kappa} g(y)-3 g(x)\right) \leq r\left(\frac{1}{\kappa} g(y)-g(x)\right) \\
& \leq r \rho(x, y) .
\end{aligned}
$$

Case (2) Whenever $g(y)<2 \kappa g(x)$, from (123),

$$
\begin{aligned}
\rho(\mathscr{T} x, \mathscr{T} y) & \leq \kappa g(\mathscr{T} x)+\kappa g(\mathscr{T} y) \\
& \leq \frac{r \eta(r)}{3 \kappa^{3}+r \eta(r)} g(x)+\frac{r \eta(r)}{3 \kappa^{3}+r \eta(r)} g(y) \\
& \leq \frac{\kappa r \eta(r)}{3 \kappa^{3}+r \eta(r)} g(x)+\frac{2 \kappa r \eta(r)}{3 \kappa^{3}+r \eta(r)} g(x) \\
& =\frac{3 \kappa r \eta(r)}{3 \kappa^{3}+r \eta(r)} g(x)=r \frac{3 \kappa \eta(r)}{3 \kappa^{3}+r \eta(r)} g(x) \\
& \leq r \rho(x, y) .
\end{aligned}
$$

Hence,

$\eta(r) \rho(x, \mathscr{T} x) \leq \kappa \rho(x, y)$ implies $\rho(\mathscr{T} x, \mathscr{T} y) \leq r \rho(x, y)$,

for all $x, y \in Z$. From (30), mapping $F_{i x}(\mathscr{T})$ is nonempty, a contradiction. Hence, $Z$ is complete.

\section{Data Availability}

No data were used to support this study.

\section{Conflicts of Interest}

The authors declare that there are no conflicts of interest regarding the publication of this paper.

\section{References}

[1] S. Cobzaş, "Fixed points and completeness in metric and in generalized metric spaces," Fundamentalnaya i Prikladnaya Matematika, vol. 22, no. 1, pp. 127-215, 2018.

[2] T. Q. Bao, S. Cobzaş, and A. Soubeyran, "Variational principles, completeness and the existence of traps in behavioral sciences," Annals of Operations Research, vol. 269, no. 1-2, pp. 53-79, 2018.

[3] T. V. An, N. V. Dung, Z. Kadelburg, and S. Radenović, "Various generalizations of metric spaces and fixed point theorems," RACSAM, vol. 109, pp. 175-198, 2015.

[4] S. Czerwik, "Contraction mappings in b-metric spaces," Acta Mathematica et Informatica Universitatis Ostraviensis, vol. 1, no. 1, pp. 5-11, 1993.

[5] S. Czerwik, K. Dlutek, and S. L. Singh, "Round-off stability of iteration procedures for operators in b-metric spaces," Journal of Nature Physical Science, vol. 11, pp. 87-94, 1997.

[6] S. Czerwik, "Nonlinear set-valued contraction mappings in b-metric spaces," Atti del Seminario Matematico e Fisico dell'Università di Modena, vol. 46, pp. 263-276, 1998.

[7] L. Ćirić, M. Abbas, M. Rajović, and B. Ali, "Suzuki type fixed point theorems for generalized multivalued mappings on a set endowed with two b-metric," Applied Mathematics and Computation, vol. 219, no. 4, pp. 1712-1723, 2012.

[8] S. L. Singh and B. Prasad, "Some coincidence theorems and stability of iterative procedures," Computers \& Mathematics with Applications, vol. 55, no. 11, pp. 2512-2520, 2008.

[9] T. V. An, L. Q. Tuyen, and N. V. Dung, "Stone-type theorem on b-metric spaces and applications," Topology and its Applications, vol. 185-186, pp. 50-64, 2015. 
[10] S. L. Singh, S. Czerwik, K. Krol, and A. Singh, "Coincidences and fixed points of hybrid contractions," Tamsui Oxford Journal of Information and Mathematical Science, vol. 24, no. 4, pp. 401-416, 2008.

[11] T. Suzuki, "Basic inequality on a b-metric space and its applications," Journal of Inequalities and Applications, vol. 2017, Article ID 256, 2017.

[12] S. Banach, "Sur les opérations dans les ensembles abstraits et leur application aux équations intégrales," Fundamenta Mathematicae, vol. 3, pp. 133-181, 1922.

[13] T. Suzuki, "A generalized Banach contraction principle that characterizes metric completeness," Proceedings of the American Mathematical Society, vol. 136, no. 5, pp. 1861-1869, 2008.

[14] R. Kannan, "Some results on fixed points," Bulletin of the Calcutta Mathematical Society, vol. 60, pp. 71-76, 1968.

[15] R. Kannan, "Some results on fixed points-II," The American Mathematical Monthly, vol. 76, no. 4, pp. 405-408, 1969.

[16] P. V. Subrahmanyam, "Completeness and fixed-points," Monatshefte Für Mathematik, vol. 80, no. 4, pp. 325-330, 1975.

[17] E. H. Connell, "Properties of fixed point spaces," Proceedings of the American Mathematical Society, vol. 10, no. 6, p. 974, 1959.

[18] M. Elekes, "On a converse to banach's fixed point theorem," Proceedings of the American Mathematical Society, vol. 137, no. 09, p. 3139, 2009.

[19] L. B. Ciric, "A generalization of banach's contraction principle," Proceedings of the American Mathematical Society, vol. 45, no. 2, pp. 267-273, 1974.

[20] S. Nadler Jr., "Multi-valued contraction mappings," Pacific Journal of Mathematics, vol. 30, no. 2, pp. 475-488, 1969.

[21] A. Amini-Harandi, "Fixed point theory for set-valued quasicontraction maps in metric spaces," Applied Mathematics Letters, vol. 24, no. 11, pp. 1791-1794, 2011.

[22] M. Kikkawa and T. Suzuki, "Three fixed point theorems for generalized contractions with constants in complete metric spaces," Nonlinear Analysis: Theory, Methods \& Applications, vol. 69, no. 9, pp. 2942-2949, 2008.

[23] M. Jovanović, Z. Kadelburg, and S. Radenović, "Common fixed point results in metric type spaces," Fixed Point Theory and Applications, vol. 2010, Article ID 978121, 2010.

[24] N. V. Dung and V. T. L. Hang, "On relaxations of contraction constants and Caristi's theorem in b-metric spaces," Journal of Fixed Point Theory and Applications, vol. 18, no. 2, pp. 267284, 2016.

[25] H. Aydi, M. F. Bota, E. Karapinar, and S. Mitrović, "A fixed point theorem for set-valued quasi-contractions in b-metric spaces," Fixed Point Theory and Applications, vol. 2012, no. 1, Article ID 88, 2012.

[26] M. A. Kutbi, E. Karapinar, J. Ahmad, and A. Azam, "Some fixed point results for multivalued mappings in b-metric spaces," Journal of Inequalities and Applications, vol. 2014, Article ID 126, 2014.

[27] H. Alolaiyan, B. Ali, and M. Abbas, "Characterization of a b-metric space completeness via the existence of a fixed point of ciric-suzuki type quasi-contractive multivalued operators and applications," Analele Universitatii "Ovidius" Constanta-Seria Matematica, vol. 27, no. 1, pp. 5-33, 2019.

[28] M. Kikkawa and T. Suzuki, "Some similarity between contractions and kannan mappings," Fixed Point Theory and Applications, vol. 2008, Article ID 649749, 9 pages, 2008.

[29] S. Reich, "Fixed points of contractive functions," Bolletino dell Unione Matematica Italiana, vol. 5, pp. 26-42, 1972.
[30] S. Park and B. E. Rhoades, "Comments on characterizations for metric completeness," Japanese Mathematics, vol. 31, no. 1, pp. 95-97, 1986. 